\title{
Electromagnetic Interference and Digital Circuits: An Initial Study of Clock Networks
}

\author{
Hongxia Wang, Samuel V. Rodriguez, Cagdas Dirik \\ and Bruce Jacob \\ Department of Electrical and Computer Engineering \\ University of Maryland, College Park, MD 20742 \\ \{hwang, samvr, cdirik, blj\}@eng.umd.edu \\ http://www.ece.umd.edu/ blj/integrity/
}

\begin{abstract}
Radio Frequency Interference (RFI) can have adverse effects on commercial electronics. Current properties of high performance integrated circuits (IC's), such as very small feature sizes, high clock frequencies, and reduced voltage levels, increase the susceptibility of these circuits to RFI, causing them to be more prone to smaller RFI levels. Besides, recent developments of mobile devices and wireless networks create a hostile electromagnetic environment for IC's. Therefore, it is important to generalize the susceptibility of IC's to RFI. In this study, we investigate the susceptibility levels and frequency ranges of RFI to the clock network of a digital device, an 8-bit ripple counter, designed and fabricated using AMI 0.5 um process technology. Our experimental setup is designed to couple a pulse modulated RF signal using the direct pin injection method. Our experiments show that relatively low levels of RFI (e.g., $16.8 \mathrm{dBm}$ delivered RF peak power with carrier frequency of $1 \mathrm{GHz}$ ) could adversely affect the normal functioning of the device under test. In the end, SPICE simulations show the sensitivity of a flip-flop, the basic building block of clock network, which further explains the experimental results.
\end{abstract}

Keywords: RFI, Pin direct injection method, Power reflection coefficient, Pulsed modulated RF, Clock network, metastability

\section{INTRODUCTION}

Numerous other structures in a computer system (e.g., external wires, cables, PCB traces, bonding interconnects, chip pins like power, ground, and data lines), can act as antennae in the presence of RFI, which is either radiated from powerful radio transmitters, wireless network devices, mobile phones and PDAs, or intentionally generated for malicious reasons. These unintentional antennae can serve to couple RF noise into the core circuit [1][2][3]. RFI that is coupled by the system can induce unwanted currents, which cause various disturbances. Also, current high performance integrated circuits (IC's), such as microprocessors, have very 
small feature sizes and are clocked at frequencies well into the GHz range while operating at reduced voltage levels. Although this has improved the ability and performance of modern systems, it has also increased their susceptibility to RFI. The electrical charge required in transistor switching decreases with relatively smaller IC feature sizes. Correspondingly, the energy required to make the device to switch is reduced, making it easier to disturb the circuit with lower RFI signal levels. As the switching speed of the IC's increases and the supply voltage scales down, the noise margin also becomes smaller. This allows external disturbances to degrade the signal integrity more easily. Even though with the advanced circuit design technology, additional circuitry design methodologies, such as offset the switching voltage, using schmidt triggering devices or bus hold circuitry, increasing the signal to noise radio, and adding input/output buffers to the circuit, could be applied to improve the noise immunity, these techniques are not generally used in the simple digital circuit systems.

Besides RF noise coupling, the inherent non-linear behavior of electronic devices in digital circuits can also cause RF signal rectification and amplification and is said to be the primary upset mechanism for integrated circuits under RFI [4]. Also, inter-modulation, cross-modulation and other disturbances are immediate effects of interference [8]. These unwanted interferences, when interpreted as or superimposed on system signals, can cause spurious state changes on logic devices and system-level breakdown [3][4].

The power levels and frequency range for which circuits are susceptible to RFI have been studied recently. Previous studies observed changes on the I-V characteristics of diodes, BJTs, and MOSFETs under RFI [1]. Susceptibility levels of a microcontroller and a DSP chip have been measured for RF interference up to $400 \mathrm{MHz}$, and data corruption was observed on the communication path between the microcontroller and RAM memory [2]. The study also showed that $20 \mathrm{dBm}$ RFI at $350 \mathrm{MHz}$ was enough to trigger the reset pin of a voltage regulator. Another study investigated the effects of RFI on the input ports of a $0.7 \mu \mathrm{m} \mathrm{CMOS} \mathrm{inverter}$ with frequency range between $20 \mathrm{MHz}$ and $1 \mathrm{GHz}$ and power levels up to $15 \mathrm{dBm}$ [3]. Dynamic failures in the form of variations in input pad propagation delay and static failures were observed when pad output signals were misinterpreted as they strayed beyond the high and low voltage logic thresholds. With devices running at higher frequencies, there is a growing concern over the electromagnetic compatibility of IC's in hostile RFI environments in wider frequency ranges, such as at and above $1 \mathrm{GHz}$. To this end, power levels and frequency ranges of RFI to which a typical synchronous high speed IC's are more sensitive, have been studied in this paper. 


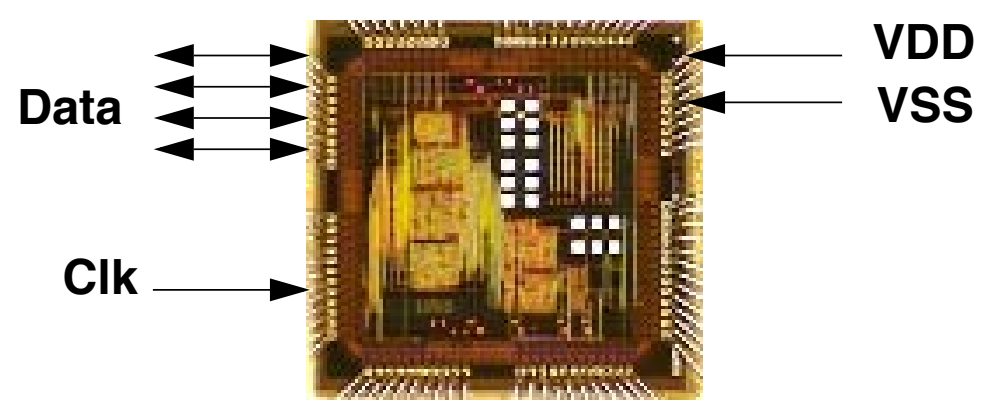

(a)

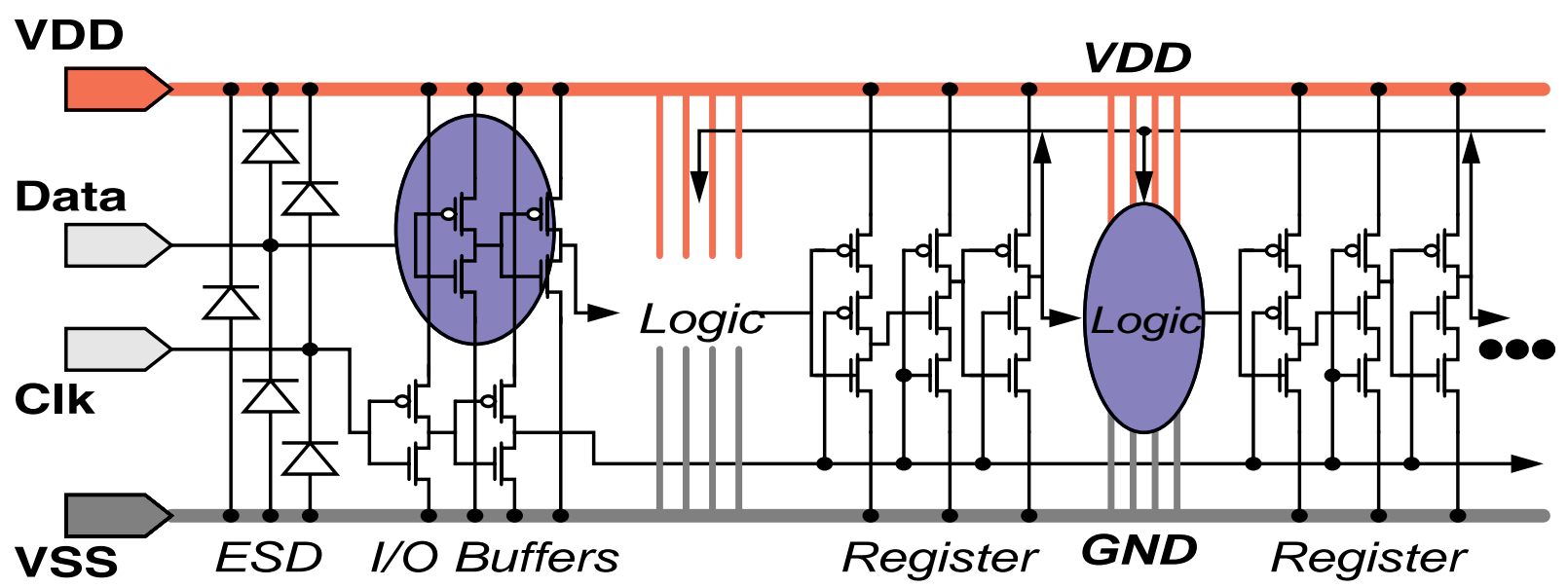

(b)

Figure 1: IC Under Test (a) The counter chip layout. (b) The logic representation.

\section{BACKGROUND - DIGITAL CIRCUITS AND THEIR VULNERABILITIES}

This work follows a system-level approach to quantify and generalize the susceptibility levels of simple digital IC's. On the system-level, signals in the basic building blocks of memory and CPU are mainly composed of the clock, power/ground, and data networks. These signals are interpreted differently under normal operation. The timing and voltage levels of the data input/output signals are important, whereas for clock signals, if it is going to drive all the flip-flops simultaneously, the rise/fall time are more critical. For power/ground planes, ground bounce is a major concern. A digital circuit with these different signals are shown in the Fig. 1. Fig. 1(a) shows the layout of the chip and Fig. 1(b) shows the internal circuitry with input ESD circuit and input buffer stages included.

The different characteristics of these signals might cause digital systems to fail in several distinct ways. Fig. 2 shows an example of metastability, the timing diagram demonstrating the roles of both data and clock signals in the latch's operation. All synchronous systems are built around pipelines of logic interspersed with some form of storage elements, such as latches. Note from Fig 2 that the transient spikes in the data input to the latch (the D signal in the diagram) do not get transmitted to the latch's output. The latch's internal state 


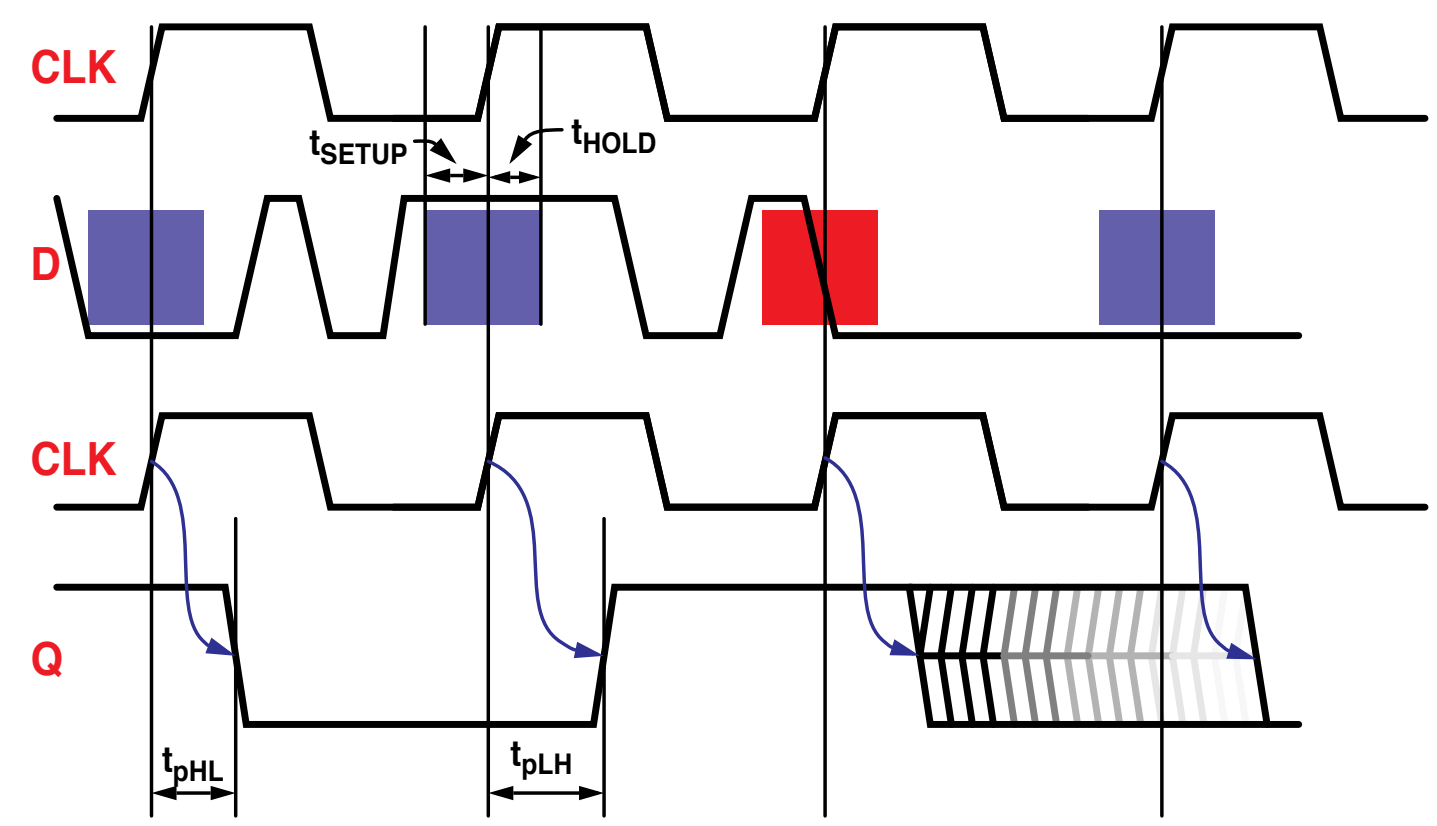

Figure 2: A Simple Digital Circuit Timing Diagram.

and thus its output will be whatever data value is seen at the latch's input during the shaded window surrounding a rising clock edge. Setup and hold time violations cause the latch to become metastable, in which the latch's state becomes undefined for an undefined length of time.

Following the discussion of timing, there are clear ways for RFI to cause a digital circuit to become corrupted since the timing of data arrival and clock edge is crucial. If a spike in the data network occurs during the latch's setup and hold time, the latch can go metastable. Similarly, if there is an RFI-induced spike in the clock network, this can send a clock edge to all latches in a complete synchronized system that occurs at the "wrong" time, possibly causing a large number of latches to misbehave simultaneously. The bottom line: if a clock edge is seen too close to a data transition, the latch's output can become undefined and remain so for a long time; therefore, if RF causes a voltage spike on the clock network, any of the latches on that clock network can go metastable. To corrupt signals on the data network, the signals must have their voltage levels changed, at the correct time, and for a long enough duration to be noticed. While for power networks, ground bounce could be a major issue that cause the system to misbehave. In this case, voltage regulators could be designed specifically to filter out these switching noises.

From the metastability example, we can see that the clock signal provide the essential control mechanism to coordinate the ebb and flow of data as it is processed, stored, or transferred through, into, or out of a digital system, this study mainly focused on the clock network. 


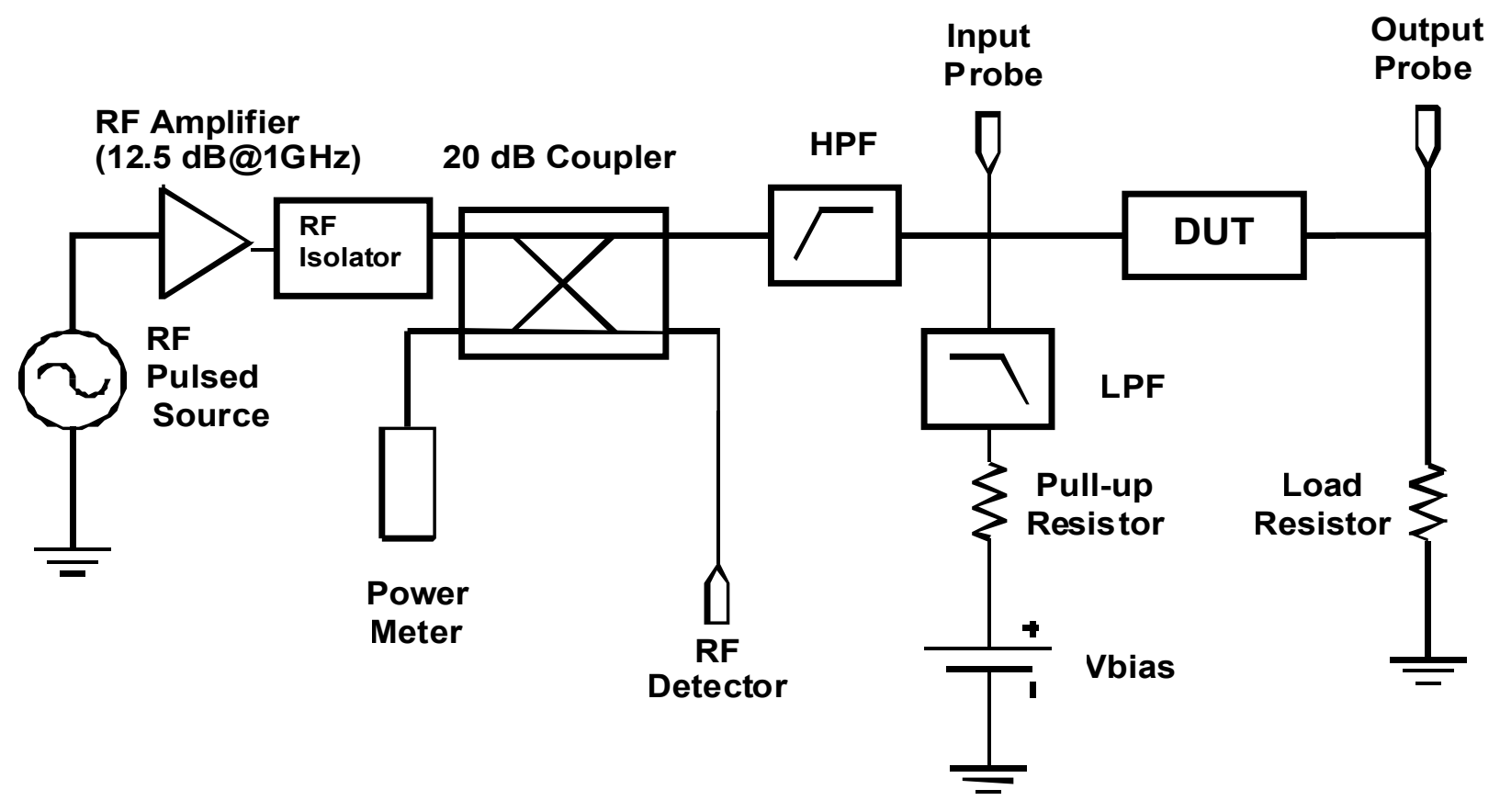

Figure 3: Experimental Setup.

\section{EXPERIMENTAL SETUP AND RESULT ANALYSIS}

To investigate the clock network, we designed the test circuit, an 8-bit ripple counter with synchronous behavior. We injected the pulse modulated RF signals, which is basically the multiplication of pulse wave with high frequency sinsoid wave, into the clock network of the testing circuit to observe the output effects. The complete experimental setup is shown in Fig. 3. The $20 \mathrm{~dB}$ coupler is used to measure the power that is delivered into the circuit and the reflected power. A bias $\mathrm{T}$ is usually used for electromagnetic coupling which is composed of a capacitor and a inductor [8]. The capacitor and the impedance of the RF injection transmission line create a single pole high pass filter. The problem is that if the value of this capacitor is too small, it will result in an AC voltage divider between the capacitor and the ESD capacitance of the input pads. The inductor and the impedance of the RF injection transmission line create a single pole low pass filter. These will cause potential loading effects. High and low pass filters are used to eliminate the possible loading effect caused by a regular bias T [7]. The 7th order butter-worth high/low pass filters with $500 \mathrm{MHz}$ and $100 \mathrm{MHz}$ cutoff frequencies respectively, are used with 50ohm input/output impedances. A $500 \mathrm{MHz}$ High Pass Filter (HPF) is used to match the RF frequency, and the $100 \mathrm{MHz}$ Low Pass Filter (LPF) is used to match the RF envelope, which depends on the pulse frequency. Bypass capacitors with the value of $0.1 \mu \mathrm{F}$ are used on the PCB board to provide closer return current paths. A $12.5 \mathrm{~dB}$ RF amplifier is employed in this setup, and the bias voltage is set to zero at the initial test measurement. In the setup above, the higher frequency component 
of the pulse modulated RF signal entered into the Device Under Test (DUT), while the lower frequency component is being measured at the input probe. Also, as we have shown in the Fig. 1, there are ESD and input buffer at each input/output pin, so the actual signal that is actually transferred through the circuit is being rectified and by the ESD component.

The DUT is a ripple counter and it is designed and fabricated using the AMI $0.5 \mu \mathrm{m}$ process technology through MOSIS. The operating clock of the ripple counter is driven by a multiplexer that chooses between an internally generated clock and an external clock, an input signal to the circuit, "EXTCLK". A separate input signal, "CLKSEL" is used as a control signal to the multiplexer. In this study, we injected pulsemodulated RF signals into the EXTCLK input pin, observed the effect on the ripple-counter output, and recorded the required RF frequency and power level to trigger the output state change.

In the normal circuit operation, the ripple counter counts according to the internal clock of the chip. Once we ensured the chip was working properly using the internal clock, we injected a pulse-modulated small RF signal, with pulse width of $10 \mu \mathrm{sec}$ and $1 \mathrm{msec}$ pulse period into the EXTCLK input pin of the chip. We observed the counter outputs. The reason for using pulsed RF instead of continuous wave is because the total energy of a pulsed RF signal is adjustable. When RF pulses are applied with a relatively low duty cycle, one percent in the setup, the total energy is much lower compared to continuous wave RF signals. This prevents the circuit from overheating and potential damage to RF signal generator and RF amplifiers caused by the reflected power. As we described earlier in the paper, the purpose of this study is first, to find out the circuit input impedance characteristic of the pins of our interests; and second, based on which to investigate the power level and frequency range to which the counter is most sensitive - i.e., those frequencies and power levels that cause output state changes. Following the experimental setup in Fig. 3, we measured the power at each port of the coupler at frequency range from $800 \mathrm{MHz}$ to $2 \mathrm{GHz}$, out of which there are no obvious state changes observed with the amplifier employed (due to the limitation of the safe operating range of the RF amplifier.

For different frequency and power levels of the injected RF signal, we observed the least significant bit of the ripple counter output. When RF pulses were coupled into the input EXTCLK pin of the counter, we noticed clear state changes at the output, as shown in Fig. 5(c), for a source frequency of $1.0 \mathrm{GHz}$.

First, we measured the input impedance of the input EXTCLK pin. Fig. 4 shows the magnitude and the phase of the input impedance by a vector network analyzer. We observed that the input impedance of the counter circuit is frequency-dependent. However, the magnitude of the impedance is independent of the injected RF power with the power applied. From Fig. 4, we can see that there are several resonant frequencies in the circuit, $0.2,0.5$, and $1.2 \mathrm{GHz}$ respectively. These resonant frequencies could be caused by the input ESD circuitries' PN junction capacitances, the circuit parasitics, PCB board traces, connectors, the inductance of 

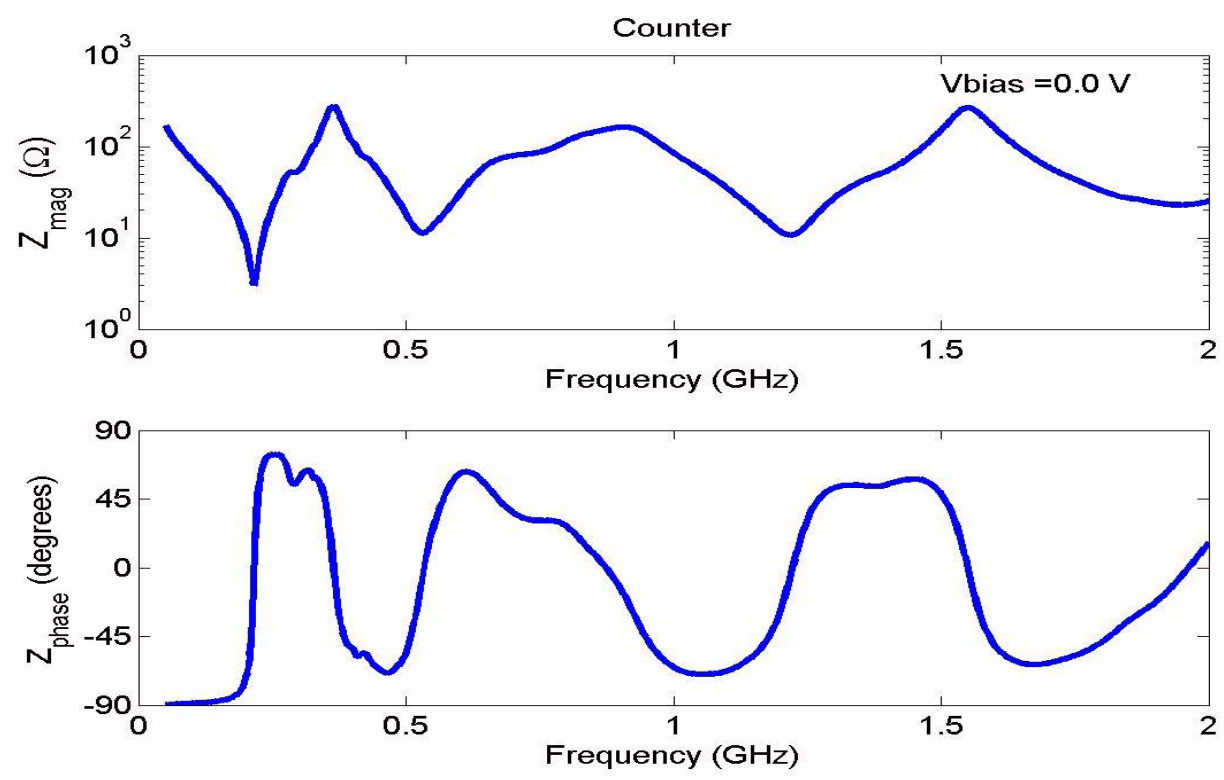

Figure 4: Input Impedance of DUT.

the wires between the die and the package (this inductance often causes a peak of high impedance in the supply at the resonance frequency of the chip capacitance and the package inductance), supply spikes [8], bonding pads and bonding wires.Since noise is mostly around the range of several hundred $\mathrm{MHz}$, we are working on shifting these resonant frequencies to higher frequency ranges by designing a new PCB board with on-board SMA connectors directly connected to the test pins and with increased on-chip capacitors to have a better matching impedance. So, in this experiment, we primarily focused on the resonant frequency that is above $1 \mathrm{GHz}$, which is around 1.2GHz. Therefore, we injected modulated RF with carrier frequencies of 1.0 $1.3 \mathrm{GHz}$ into the external clock pin. The pulse width and pulse period remained the same. We observed output state changes which demonstrated that the input voltage was high enough to trigger the counter output to switch. Table. 1 summarizes the RF power levels that are required to make such state changes. The table includes the injected power; the reflected power caused by the mismatched input impedance; and the power that was actually delivered into the pin. The last row in the table is the power-reflection coefficient $\Gamma$, which is defined as square root of the reflected power over injected power. The frequency which the circuit is most susceptible (i.e., that which requires the least power to make a state change, $1.2 \mathrm{GHz}$ with the frequency range we tested), coincides with the lowest power-reflection coefficient. This coefficient is less than half than those at other frequency ranges, as we can see from the Table 1 . This is caused by the input circuitry resonant frequency, 1.2 GHz from Fig. 4, at which less power is required to make a state change.

We also recorded the output behavior right around the threshold power levels. The output shows full state changes, partial state changes, and oscillations shown in the Fig. 5. In Fig. 5, the input, RF envelope, and output voltage waveforms at RF carrier frequency of $1 \mathrm{GHz}$ with pulse width $10 \mu \mathrm{s}$ and pulse period $1 \mathrm{~ms}$ are 
Table 1: Power required to make state changes $(\mathrm{dBm})$

\begin{tabular}{lllll}
\hline Frequency $(\mathrm{GHz}):$ & $\mathbf{1 . 0}$ & $\mathbf{1 . 1}$ & $\mathbf{1 . 2}$ & $\mathbf{1 . 3}$ \\
\hline \hline Injected Power $(\mathrm{dBm}):$ & 20.1 & 17.9 & 16.9 & 20.5 \\
\hline Reflected Power $(\mathrm{dBm}):$ & 13.3 & 13.8 & 6.3 & 15.3 \\
\hline Delivered Power $(\mathrm{dBm}):$ & 16.8 & 15.2 & 14.7 & 16.6 \\
\hline $\begin{array}{l}\text { Power Reflection } \\
\text { Coefficient } \Gamma\end{array}$ & 0.56 & 0.65 & 0.39 & 0.65 \\
\hline
\end{tabular}

shown. In Fig. 5 (c), the circuit exhibits output state changes when a relatively small amount of RF power, 20.5 $\mathrm{dBm}$, is injected. At this point, the induced input voltage to the circuit is higher than the device threshold voltage, therefore triggers the counter output to a complete switch. This is due to the rectification and the amplification of the RF caused by the junction of the diode in the ESD protection circuit [4][5]. The ESD rectification amplifies the input signal seen at the pin and induces DC voltages on the P-N junctions of ESD protection circuit. If this induced DC voltage is higher than the threshold voltage of the circuit, injected pulse modulated RF envelope is interpreted as a valid signal and causes a state change at the outputs. In Fig. 5(a), when the induced DC voltage is below the threshold voltage of the circuit,with $18.1 \mathrm{dBm}$ injected RF power, there is not enough input voltage to trigger the output to make a state change. In Fig. 5(b), we increased the RF level to $20.1 \mathrm{dBm}$, so that the induced DC voltage at the input pin is close to the device threshold voltage. When the induced DC voltage is right around the threshold voltage, the noises from different resources, such as power supplies and cables, are coupled with the input, and play a major role in the switching activities of the output. These noise spikes superimposed on the top of the input, triggering the circuit output to switch back and forth causing oscillations.

\section{SPICE SIMULATION RESULTS}

In the previous section, we had shown how the circuit behaved when we injected the external clock pin with 1GHz pulsed modulated RF. Fig. 5(a) shows no output change behavior at all. The graphs Fig.5 (b) and (c) have different output switching behavior and therefore raise questions about what is causing the behavior. For instance, Fig. 5 (c) shows the circuit responding only to the envelope of the RF (the leading edge). Is this because the RF frequency is too high for the circuit to respond, or is the RF frequency able to drive the circuit, but the circuit is put into metastability and requires a correctly-timed clock edge to be driven out? Similarly, 


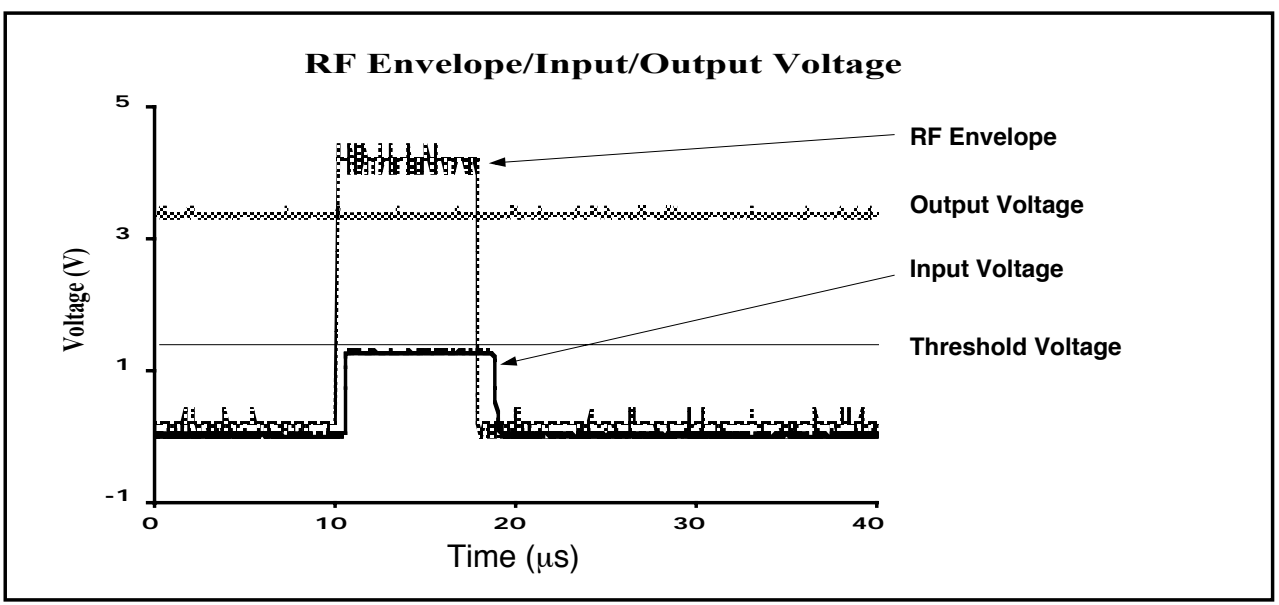

(a)

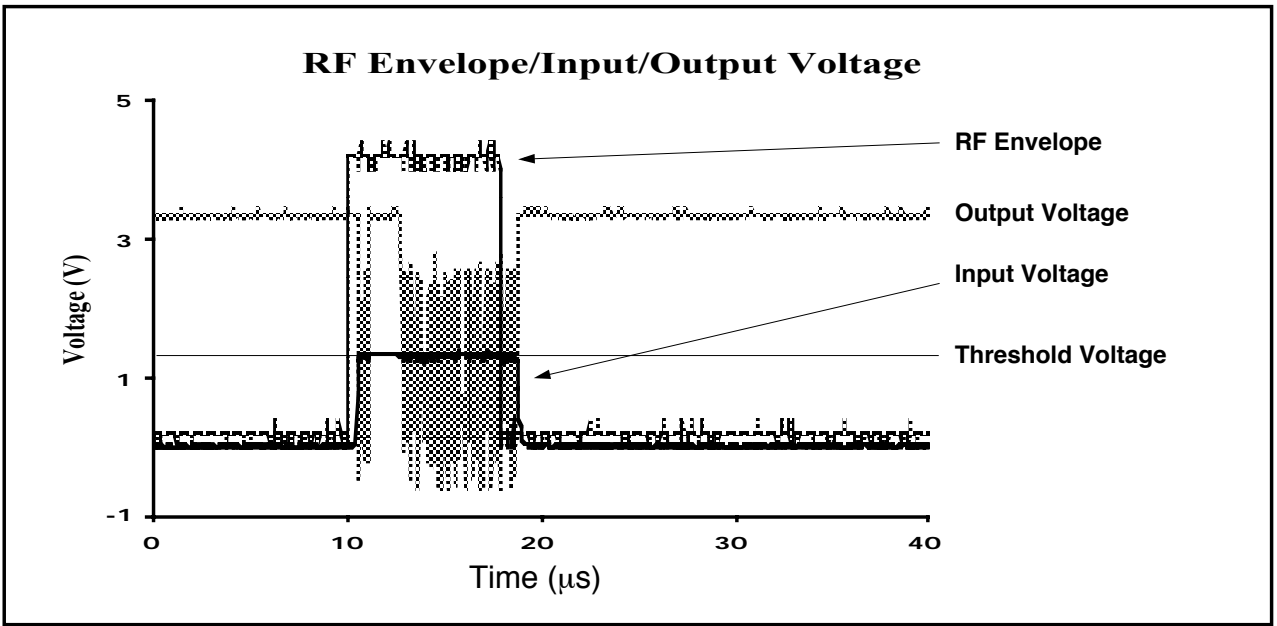

(b)

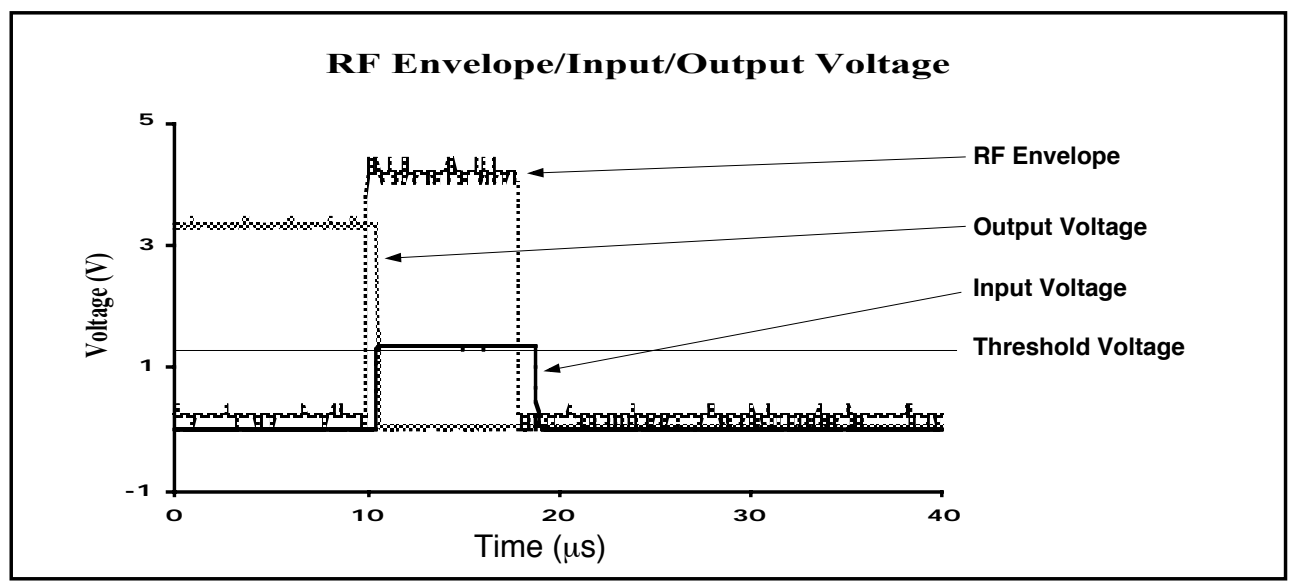

(c)

Figure 5: Rectification of RF causing output state changes. (a) Induced input voltage is below threshold voltage; no state changes observed at the output; (b) Induced input voltage is right around threshold voltage and the output to change back and forth; (c) Induced input voltage is above threshold voltage; single change at the output is observed. Note the horizontal line indicating threshold voltage is exaggerated slightly (the minute differences are not visible at this scale). 
Fig. 5(b) shows the circuit responding to much more than just the envelope of the RF: Is it responding to the noise on top of the clock signal? or to the actual RF signal that went through the circuit? or to the input resonant frequencies? Also, note that the frequency of the output response in Fig. 5(b) is much less than the frequency of the RF input. Does this suggest that one might be able to rule out the RF signal as the cause for the behavior (leaving noise as the most likely explanation)? With all these doubts in mind, we performed some SPICE simulations to try to understand these mechanisms, answer these questions, and rule out one or more causes.

Since our DUT is basically a series of cascaded flip-flops, we choose a single filp-flop as our SPICE simulation device and excited it with different RF frequencies. Fig. 6 (a) shows the simulated D flip-flop circuit configured as a toggle flip-flop. In this configuration, the output Q should change state whenever a rising edge is seen at the CLK input. Fig. 6(b) shows the internal circuit configuration of the flip-flop. To replicate the experimental setup, we used the transistor SPICE parameters corresponding to the AMI 0.5 $\mu \mathrm{m}$ fabrication process that was used to fabricate our DUT in addition with the parasitics extracted from the layout without the ESD pad information included. We excited the circuit with a range of clock frequencies and observed the outputs. Predictably, the flip-flop deviates from its normal behavior and produces incorrect outputs after a certain clock frequency is reached. This happened when the period between clock rising edges is too small to allow the flip-flop output to propagate back through the flip-flop completely. Fig. 7 shows the four waveforms at CLK, Q, D1 and D2 in Fig. 6(b) respectively. The signal D1 (third waveform from the top) corresponds to the voltage at node D1 shown in Fig. 6(b), and the signal D2 (bottom waveform) corresponds to the voltage at node D2 also shown in Fig. 6(b).

The waveforms of Fig. 7 provides insights on the different failure modes observed for different clock frequencies. These waveforms show that the main explanation to the different fault modes seen at the output is the amount of time given for internal waveforms to propagate through the flip-flop. Fig. 7 (a) shows results with clock frequency of $1 \mathrm{GHz}$; it produces periodic output that is $1 / 6$ of the input clock as opposed to $1 / 1$ ratio (shown as the Q waveform below the topmost CLK waveform). In this instance, the latches that make up the flip-flop are driven into meta-stability on every $6 \mathrm{~ns}$ and sixth cycle of the clock. The first latch, in a logic-low state (see the D2 signal), is driven to meta-stability with the falling edge of the RF and recovers in a logic-high state on the next falling edge of the RF. The next falling edge of the RF signal causes it to go meta-stable again, from which it does not completely recover for three more cycles. The behavior results in a 6:1 ratio of RF/ clock input frequency to the output frequency. This behavior is functionally similar to exciting a lowfrequency resonance in the circuit with a high-frequency input and could be the potential cause of the behavior in Fig. 5(b).

Fig. 7 (b) shows waveforms with higher clock frequency, $1.4 \mathrm{GHz}$, and also produces unexpected outputs. But unlike the first output waveform, the output in this case is transient and only one pulse is 
produced. Fig. 5(c) shows the counter circuit responding initially to the RFI, but only for a single state-change which is corresponding to Fig. 7(b) suggesting again that RF reaching the circuit could be the cause of the observed output. Fig. 7(b) shows that the internal latch (D2) fails to recover from the second metastable instance. The internal latch is driven from a mid-point logic value into metastability and ultimately falls to logic-low. Fig. 7(c) shows an even simpler scenario with frequency of $1.8 \mathrm{GHz}$, one in which the first hint of a clock signal (RF) changes the state of the circuit, but the RF cycle time is too short for the inverters to switch completely, resulting in the familiar saw-tooth waveform in D1. These two graphs show that the behavior in Fig. 5(c) could be caused by high-frequency RF reaching the circuit (frequencies too high for the circuit to respond) high enough to make the circuit difficult to come out of metastability.

Fig. 5(b) could be easily seen as potentially caused by noise on top of the input signal(note that the brief period of logic-high during the window of output corresponds to a brief window of lessened noise in the Input Voltage signal). However, we can't exclude the possibility that the output could also be caused by the regular RF input forcing the circuit to behave in an irregular manner. Fig. 7(d) shows an example of a continuous-wave clock input fed into the flip-flop, with frequency of $1.355 \mathrm{GHz}$, yielding an aperiodic output with two pulse train. One interesting thing to point out is that the clock frequencies that generate aperiodic output and periodic output differ by only $2 \mathrm{MHz}$, which means that at $1.333 \mathrm{GHz}$, the output is periodic, similar to the $1 \mathrm{GHz}$ one; while at $1.335 \mathrm{GHz}$, we observed the aperiodic pulses. This behavior shows that with the increasing of the clock frequency, the output failure modes change from periodic, to aperiodic, to barely triggering the output with one pulse and finally to not triggering the output at all.

In all, while signal noise could very well cause the behavior in Fig. 5(b), it could just as easily be the regular RF itself combined with these irregularity shown in Fig. 7(d). In summary, it is not clear whether the input noise, a (possibly rectified) periodic RF signal passes all the way to the circuit combined with aperiodic output shown as Fig. 7(d) or the behavior shown in the Fig. 7(a) cause the behavior seen in Fig. 5(b). If the RF is successfully filtered out by the ESD circuitry, leaving a simple DC bias, then noise on that signal could be the major cause the observed behavior.

These simulations provide intuitive views of our experimental results. In the future work, we will address these questions in more detail by investigating the counter circuit behaviors under different frequency ranges in the physical experiment setup we used. 

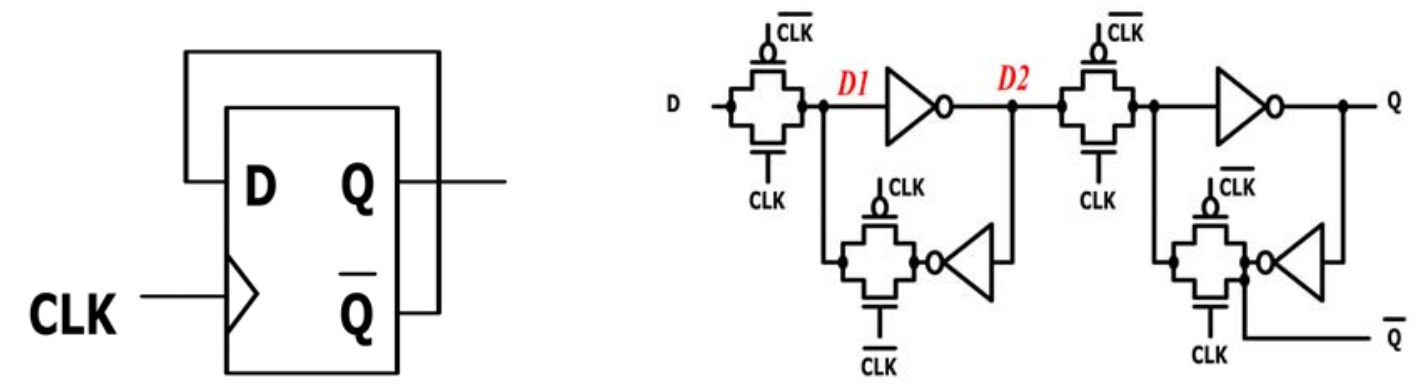

Figure 6: D Type Filp-flop. (a) D flip-flop circuit configured to change output state at every CLK edge. (b) The internal circuit configuration used for this flip-flop, which uses master-slave latches.

\section{CONCLUSION AND FUTURE WORK}

In this study we have shown that relatively low levels of pulse-modulated RFI signal can affect the normal functioning of a simple digital counter circuit clock network by pin direct injection method and SPICE simulation results were demonstrated to support our experimental results. More experiments will be done to further investigate other parameters, such as RF pulse width, pulse rise/fall time, input bias voltage, supply voltage's effects on the power level required to make state changes at the output. Additionally, we plan to test the susceptibility levels of data input/output and power/ground networks as compared to the clock network. A new PCB board is being developed to accurately match the input impedance and to shifting the resonant frequencies to a higher range. In the future study, we are planning to test the chip over a wider frequency ranges using higher power amplifiers in the future.

\section{ACKNOWLEDGEMENTS}

Special thanks to Dr. John Rodgers and Mr. Todd Firestone at The University of Maryland, Electrical and Computer Engineering department, for their tremendous help on experimental setups and analysis of the results, which made this work possible. The work of Hongxia Wang and Samuel Rodriguez is supported by the Laboratory of Physical Sciences in College Park, MD. The work of Cagdas Dirik is supported in part by DOD MURI award AFOSR-F496200110374. The work of Bruce Jacob is supported in part by NSF CAREER Award CCR-9983618, NSF grant EIA-9806645, NSF grant EIA-0000439, DOD MURI award AFOSRF496200110374, the Laboratory of Physical Sciences in College Park, MD, and by Cray. 


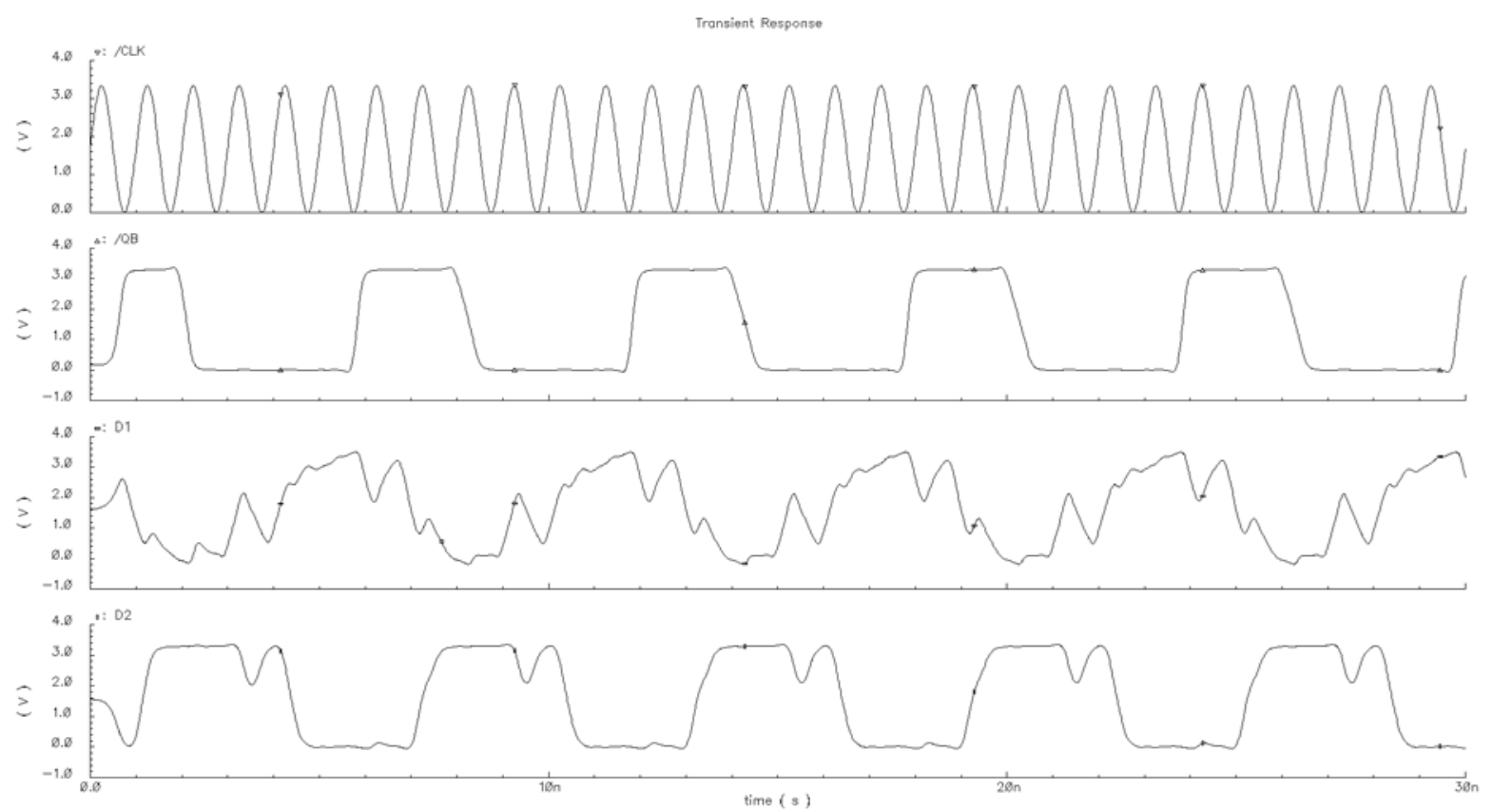

(a)

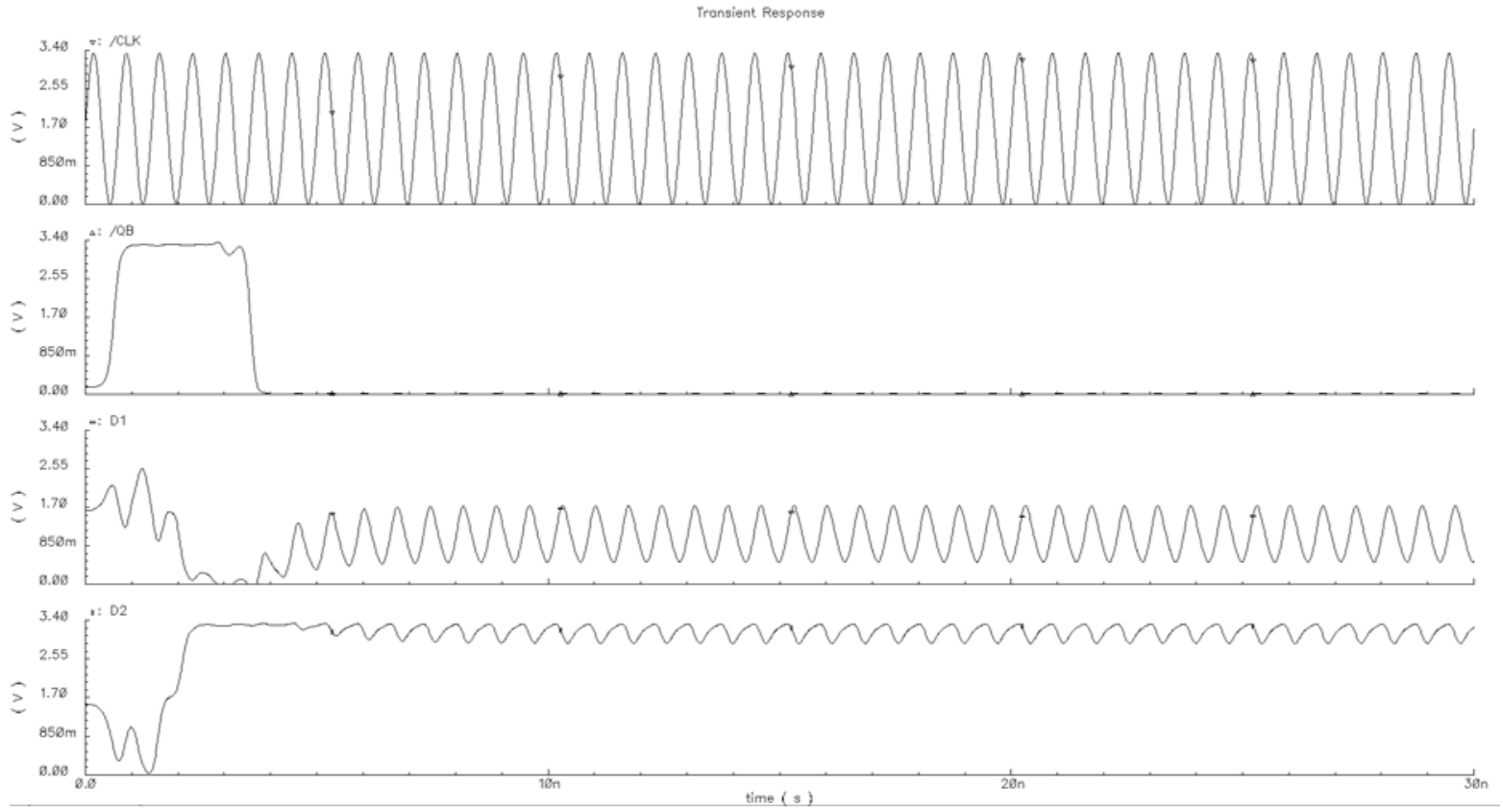

(b) 


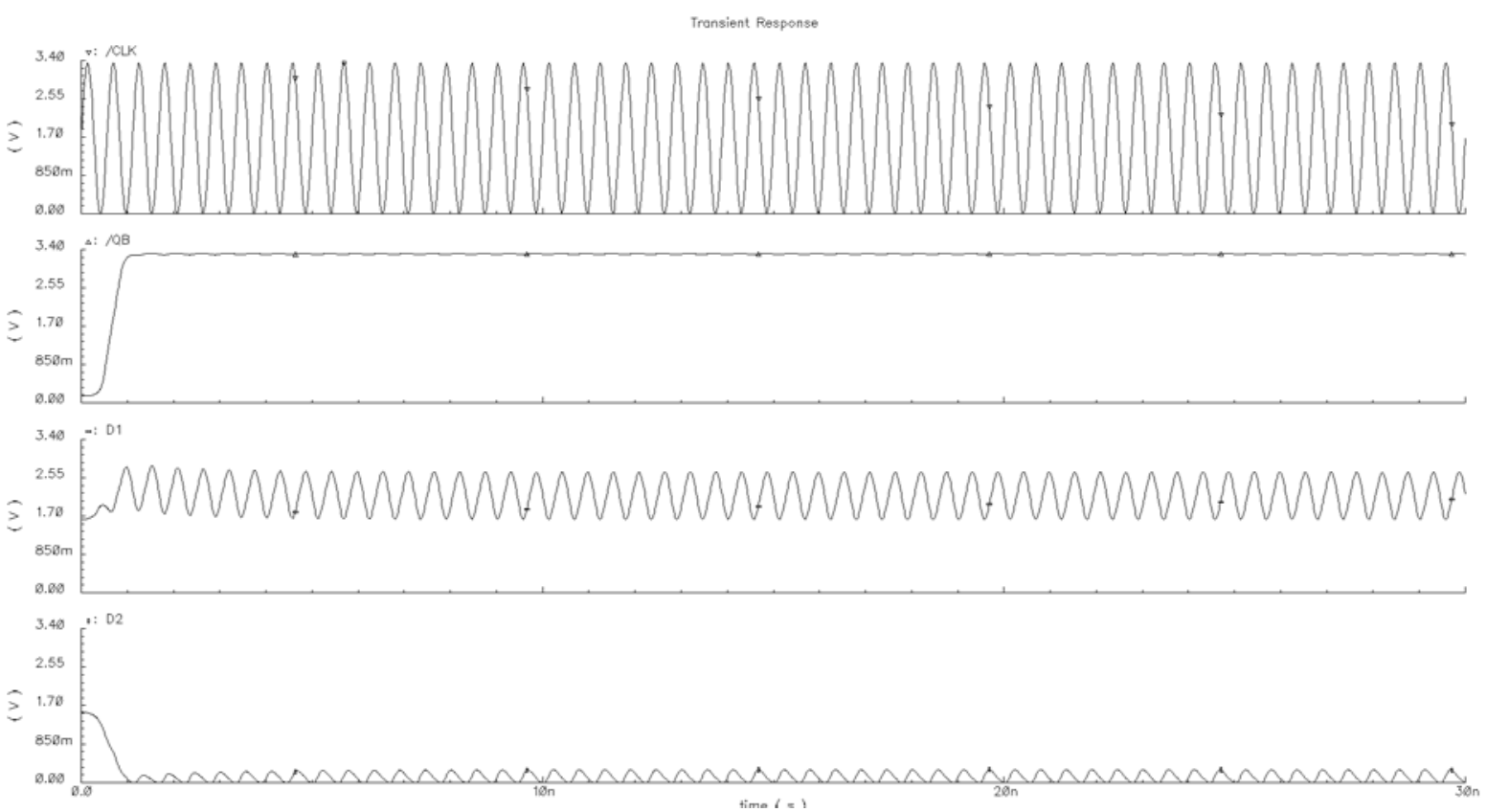

(c)
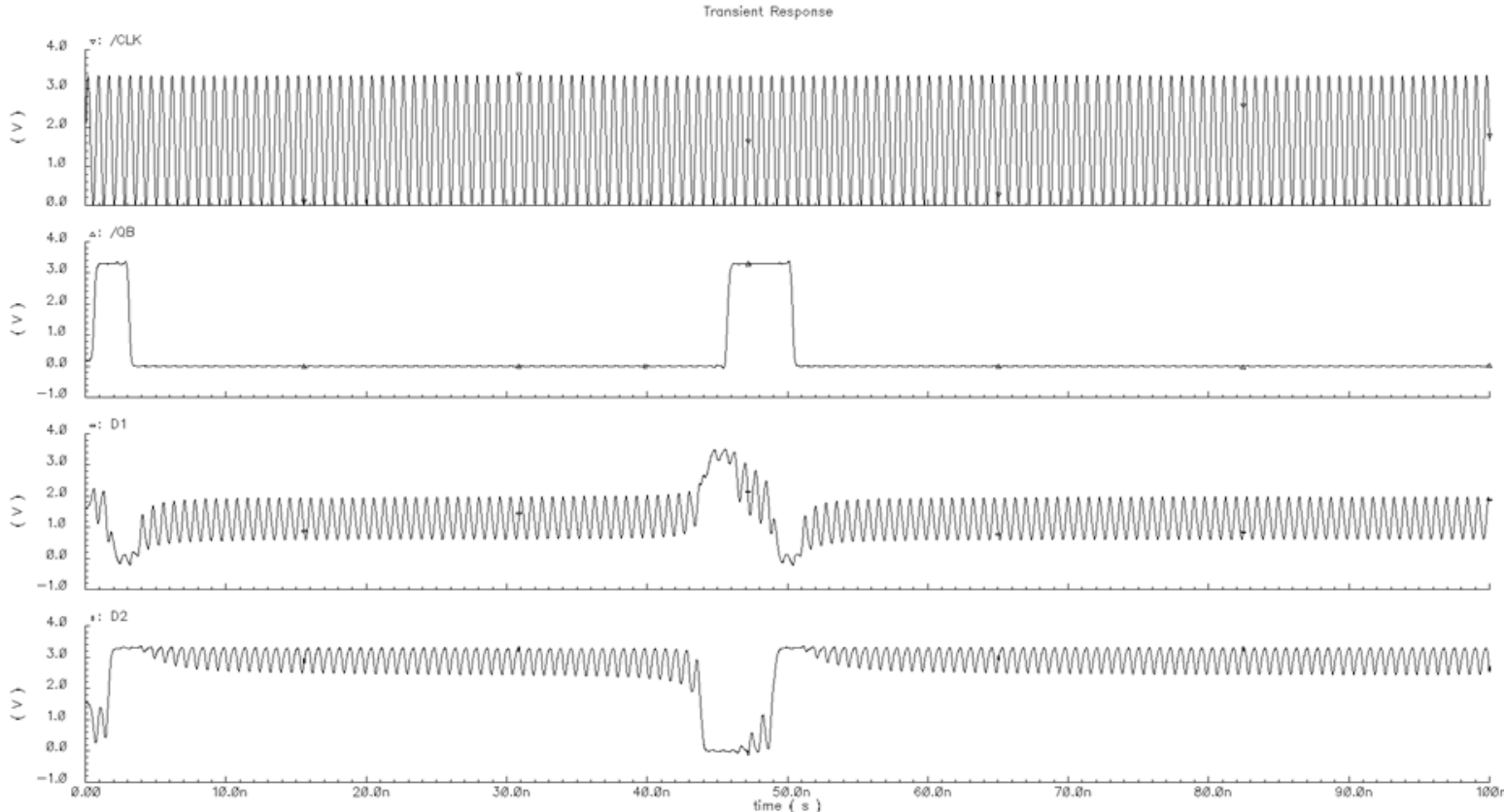

(d)

Figure 7: Output waveforms using different clock frequencies. The top waveform shows the clock signal. The next waveform is the flip-flop Q output. The next two waveforms are the internal flip-flop nodes D1 and D2. (a) Incorrect periodic output, (b) Incorrect transient output with single state changes, (c) Incorrect output without state change. (d) Incorrect aperiodic output. 


\section{REFERENCES}

[1] F. Fiori, "Integrated Circuit Susceptibility to Conducted RF Interference", Compliance Engineering 17, no. 8 (2000), pp. 40-49.

[2] S. Baffreau, S. Bendhia, M. Ramdani, E.Sicard, "Characterisation of Microcontroller Susceptibility to Radio Frequency Interference", Proc. of the Fourth IEEE International Caracas Conference on Devices, Circuits and Systems, 17-19 April 2002, pp. I031-1 -I031-5.

[3] F. Fiori, S. Benelli, G. Gaidano, V. Pozzolo, "Investigation on VLSI's Input Ports Susceptibility to Conducted RF Interference", IEEE International Symposium on Electromagnetic Compatibility, 1822 Aug. 1997, pp. $326-329$.

[4] D. J. Kenneally, D.S. Koellen, S. Epshtein, "RF Upset Susceptibility of CMOS and Low Power Schottky D-Type, Flip-Flops", IEEE National Symposium on Electromagnetic Compatibility, 23-25 May 1989, pp. $190-195$.

[5] J. Rodgers, T. M. Firestone, V. L. Granatstein, "Nonlinear Effects in Advanced Communication Circuits Excited by Pulsed RF", http://www.ireap.umd.edu/MURI-2001/Review_14Nov03/ 10_rodgers.pdf.

[6] J. G. Tront, "Predicting URF Upset of MOSEFET Digital I C's", IEEE Transactions on Electromagnetic Compatibility, Volume, EMC-27, Mo.2, May 1985.

[7] Todd Firestone, "RF Induced Non-Linear Effects in High-Speed Electronics", Thesis, University of Maryland, College Park, 2004.

[8] E. Sicard, C. Marot, J. Y. Fourniols, M. Randani, "Electromagnetic Compatibility for Integrated Circuits", Contribution to book IEEE Review of radio Sciences 1998-2001 URSI, IEEE Press-ISBN 0471-26866-6, 2002. 